\title{
RESERCH ON URBAN SPATIAL EXPANSION MODEL BASED ON MULTI -OBJECT GRAY DECISION-MAKING AND CA :A CASE STUDY OF PIDU DISTRICT, CHENGDU CITY
}

\author{
LIU Zhichen ${ }^{1,2}$ LI Ying ${ }^{1,2 *}$ \\ ${ }^{1}$ Navigation College, Dalian Maritime University, Dalian 116026, China \\ ${ }^{2}$ Environmental Information Institute,Dalian Maritime University, Dalian 116026, China
}

KEY WORDS:CA,Multi - Object Gray Decision-making,Urban Spatial Expansion,Pidu District

\begin{abstract}
:
This paper from the perspective of the Neighbor cellular space, Proposed a new urban space expansion model based on a new multiobjective gray decision and CA. The model solved the traditional cellular automata conversion rules is difficult to meet the needs of the inner space-time analysis of urban changes and to overcome the problem of uncertainty in the combination of urban drivers and urban cellular automata. At the same time, the study takes Pidu District as a research area and carries out urban spatial simulation prediction and analysis, and draws the following conclusions:(1) The design idea of the urban spatial expansion model proposed in this paper is that the urban driving factor and the neighborhood function are tightly coupled by the multi-objective grey decision method based on geographical conditions. The simulation results show that the simulation error of urban spatial expansion is less than $5.27 \%$. The Kappa coefficient is 0.84.It shows that the model can better capture the inner transformation mechanism of the city. (2)We made a simulation prediction for Pidu District of Chengdu by discussing Pidu District of Chengdu as a system instance.In this way, we analyzed the urban growth tendency of this area.presenting a contiguous increasing mode, which is called "urban intensive development".This expansion mode accorded with sustainable development theory and the ecological urbanization design theory.
\end{abstract}

\section{INTRODUCTION}

Urban expansion has an important impact on social and economic stability, ecosystem balance, and social equity, and will continue to be one of the most important global issues affecting humanity in the 21 st century. Urban expansion is a series of cumbersome process of historical space evolution. It has self-organizing features and is also subject to external macro factors. Since the second half of the 20th century, with the popularization of highperformance computers, the analysis and processing of massive data has become a reality,Geographic Information System (GIS) technology has matured and integrated with it, bringing great opportunities for computer simulation of urban space models. They use large-scale GIS software to store spatial and attribute data in urban spatial databases, rely on powerful spatial analysis functions to make management decisions for urban extension systems, and integrate relevant mathematical models, Such as DUEM model, SLEUTH model, High-resolution St.Lucia model, ANN-CA model, UrbanSim model and so on, Achieved better simulation results. In 1996, Xie Yichun used ArcView as a platform to integrate the Urban Dynamic Evolutionary Model (DUEM). This model fully considered the complexity and dynamics of the city, and finally used the Buffalo suburb of New York as a system example to simulate predictions. Verify the feasibility of the system. This is the first systematic application of GIS and CA in urban extension studies. Since the 1990s, WuFulong (1998) organically integrated the cellular automata model and the multi-factor assessment model (MCE), applied AML and $\mathrm{C}$ language in Arc/Info, and implemented GIS on a unified interface. The integration of the CA model and the MCE model under this platform enabled the simulation of urban land expansion in Guangzhou, Guangdong Province, China. In 2002, Waddell, a professor of urban simulation and policy analysis at the University of Washington, used java development language to develop a multi-agent model and developed a Urban Simulation UrbanSim model system. The model system mainly has the functions of urban growth management, urban land planning and traffic policy analysis. In 2004, Benenson and Torrens collaborated to build the first object-oriented geography automaton prototype simulation system OBEUS, which is used to establish the dynamic change simulation of living environment based on real geographic space . Immediately, domestic scholars continued to inherit the research experience of the predecessors, integrated development of urban expansion systems, and achieved certain results with the urban expansion system based on the CA model development and design, combined with different mathematical methods such as ant colony algorithm, multi-criteria Judgments, genetic algorithms, and artificial neural networks, etc., have created an urban expansion simulation system that can better analyze the evolution rules of urban space, making the research on integrated development of urban spatial expansion systems based on the CA model increasingly perfect. $\mathrm{Li}$ Xia, Ye Jiaan, Liu Xiaoping and others developed the Geographical Simulation and Optimization System (GeoSOS), which is well-suited for urban expansion prediction. Therefore, exploring the laws of urban expansion and innovating a CA model that can better analyze the evolution of urban space will make the study of urban spatial evolution based on CA increasingly perfect, which is of great significance for promoting the sustainable development of cities. 
Based on the above situation, this paper establishes a complete model of urban spatial expansion through the multi-objective grey decision-making theory and CA principle, and conducts a research on the Pidu District of Chengdu.

\section{URBAN SPATIAL EXPANSION MODEL BASED ON MULTI-OBJECTIVE GREY DECISION}

\subsection{Research area overview and data processing}

In Pidu County of Chengdu District, the county area was formally withdrawn on January 22, 2017. It was established as a district of Chengdu City. It is a modern rural ecological satellite city with geographical coordinates between longitude $103^{\circ} 42^{\prime} \sim 104^{\circ} 2$. 'Between $30^{\circ} 43^{\prime} \mathrm{N}$ and $30^{\circ} 52^{\prime} \mathrm{N}$, due to the completeness and specificity of data acquisition, the entire area of this district was selected from the entire area of Huadu Town, Youai Town, Deyuan Town and Gaoxin West District. The district area of pIdu District is 438 square kilometers, and the construction area is 56 square kilometers. The population of the registered population in the study area is about 558,000. In the past two decades, the city has undergone dramatic changes and has a certain value.

There are four types of data required for the urban spatial expansion model: urban land use status data, urban traffic planning data, slope data, and non-construction data. The three years of 1998, 2005, and 2012 were selected as the time series for the calculation of the operation. The specific sources of model operation simulation materials are "Chengdu Statistical Yearbook" (1998-2015), "Chengdu Urban Land Utilization Master Plan (2006-2020)", "Chengdu Urban Master Plan (20112020)" and "Chengdu The twelfth five-year plan for national economic and social development, etc.

According to the purpose of this study and the characteristics of the natural and geographical environment in the Pidu district, reference is made to GB/T 21010-2007 "Classification of Land Use Status,"and ENVI software is used for remote sensing imagery for land classification. And convert the data to grid format for model reading. The results of land use maps in 1998, 2005, and 2012 are shown in Figure 1, Figure 2, and Figure 3. The traffic grading layers are shown in Fig. 5, which are the current traffic layers of 1998, 2005, and 2012, and the data layers buffered by grades. In the data extraction, the buffer zone of the traffic line is defined as three levels of $0-120 \mathrm{~m}$ for the traffic level one, $120-300 \mathrm{~m}$ for the traffic level two, and $300-650 \mathrm{~m}$ for the traffic level three. The three grades represent the difference in the distance between the main roads of Pidu and the extension of urban construction land. Slope data is shown in Figure 4. Finally, the data of non-constructed area and city center are extracted. the non-constructed area data in the study is layered by the basic ecological control line in the cutting research area of ArcGIS, mainly including rivers, scenic spots for tourism, special land for government use. With land reserved for nature, the type of land used in the area has long-term stability and characteristics. This data is modeled in a separate layer to participate in the calculation of the model. The area covered by this layer does not change before and after the simulation. The purpose of this data is to reduce the "produced by the government's subjective behavior on the expansion of urban space during the operation of the model". And under the influence. The second is the distance from the city center. According to the area of Pidu District, with reference to previous studies, it is determined that urbanization is the most likely within a distance of 300 meters from the center of the city, and the impact of urban centers on the expansion of the city will be greater between 300-1000 meters. The smaller the size, the greater than $1000 \mathrm{~m}$, has little effect on the urban expansion.

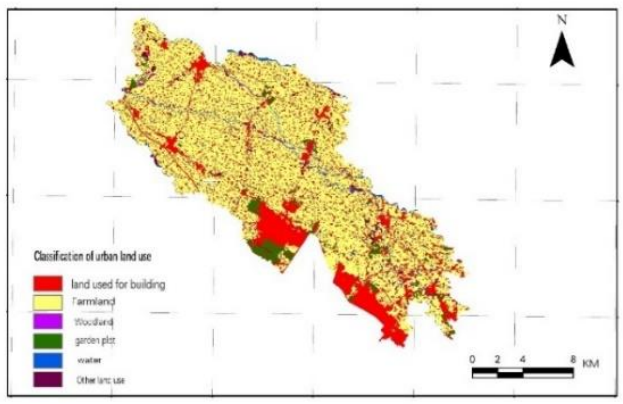

Figure 1 Classification of Land Types in 1998

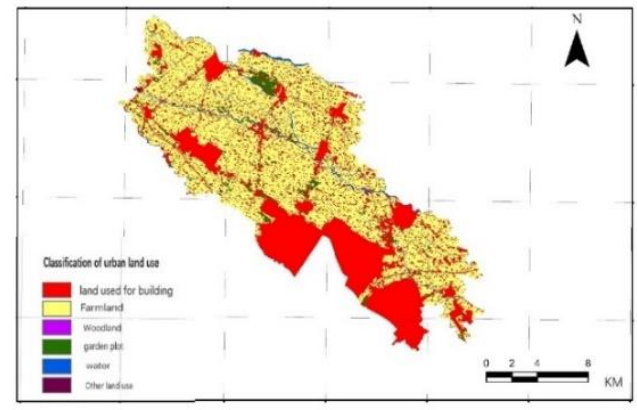

Figure 2 Classification of Land Types in 2005

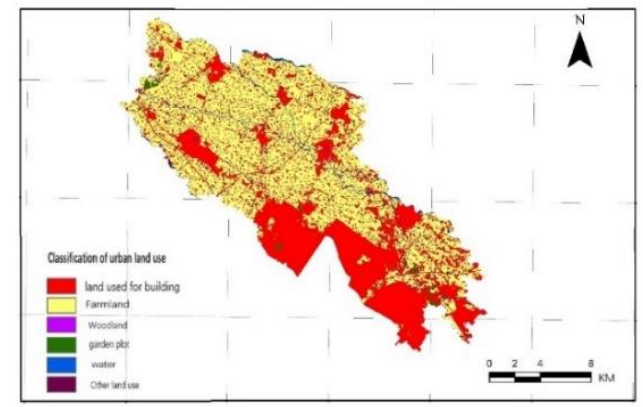

Figure 3 Land Type Classification Results for 2012

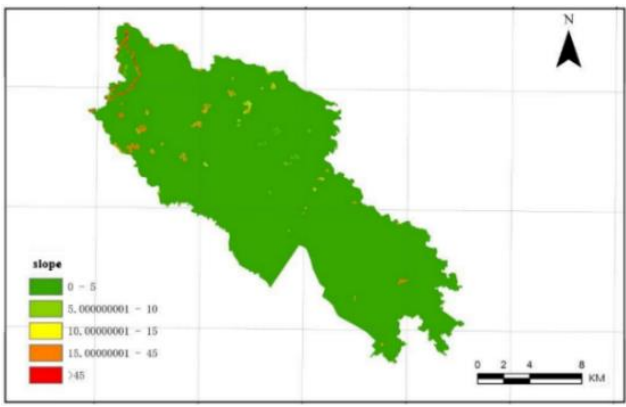

Figure 4 Pidu slope map 


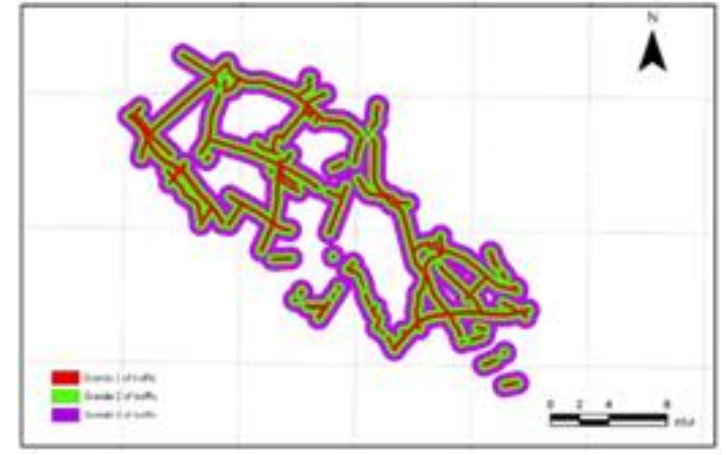

Figure 5 Pidu District Traffic Layer

\subsection{Simulation prediction experiment}

This study adopts the mathematical method of multi-objective grey decision-making, integrates the transformation rules of urban spatial expansion model into the driving mechanism of urban expansion, and makes the setting of parameters more ideal. The model is affected by the four goals of urban driving factors: urban agglomeration, urban land suitability, traffic accessibility, and distance from urban centers. In addition, one constraint condition (mainly influenced by ecological control lines in the study area) is established: No construction area. Urban construction areas are rasterized in a monotonically increasing manner within the $[0,1]$ interval.

During the experimental process, the urban land use status data model is the main basis for the operation of the model, and a certain urban space expansion is made. The dynamic evolution of urban land is caused by the interaction between different land types. The model is set as the target one (city concentration Intercompetition; Because the land for urban construction is largely constrained by topographic factors, urban construction can only be spread and expanded if it is less than the critical slope value. Therefore, objective 2 is set as the competition between different types of land under land suitability; The urban traffic planning data, as the influencing factor in the urban spatial expansion model, controls the behavior of urban change, and is set as the target 3 in the model (competition among different types of traffic in the degree of traffic accessibility), and this layer is also a dynamic map that has been constantly changing and changing. Floor; Non-construction data is generally made up of areas with certain characteristics or other functional planning. This part will ignore all calculation results and restrict the expansion of urban space to some extent. The data of the city center will expand its radiation to future cities. In the model set to goal four (competition with distance constraints from the built-up area of the city center)

(1) Goal 1: Competition among different types of cities under urban agglomeration

The degree of agglomeration of the cells can reflect the benefits of land agglomeration. The land agglomeration effect of a city can precisely express the possibility of converting land types into urban land.In the urban clustering degree, the competitiveness of different types of land is generally quantified using the number of cells.Through the calculation of the decision-making target judgment matrix 1 and the upper-limit effect measure formula, the 1 formula is used to calculate the value of the neighborhood state of the neighboring cells on the land concentration degree in each situation and draws four land types on the land. The higher the score under the degree of agglomeration, the higher the score, the better the agglomeration effect of urban construction land of the central cell, and the greater the possibility of conversion into urban construction land.

$$
\left.r^{(1)}=\begin{array}{cccc}
u_{11}^{(1)} & u_{12}^{(1)} & u_{13}^{(1)} & u_{14}^{(1)} \\
u_{21}^{(1)} & u_{22}^{(1)} & u_{23}^{(1)} & u_{24}^{(1)} \\
u_{31}^{(1)} & u_{32}^{(1)} & u_{33}^{(1)} & u_{34}^{(1)} \\
\vdots & \vdots & \vdots & \vdots \\
\left\lfloor u_{i 1}^{(1)}\right. & u_{i 2}^{(1)} & u_{i 3}^{(1)} & u_{i 4}^{(1)}
\end{array}\right\rfloor
$$

Where $u_{\mathrm{n} 1}^{(1)}$ represents the land concentration score of the nth cell whose neighbor (molar neighborhood) is a city construction land, and $u_{\mathrm{n} 2}^{(1)}$ represents the $\mathrm{nth}$ cell. If the neighborhood (Molar Neighbourhood) is the land concentration degree corresponding to the cultivated land, $u_{\mathrm{n} 3}^{(1)}$ represents the land concentration degree score of the $n$th cell whose neighbor (Molar Neighbourhood) is the woodland. $u_{\mathrm{n} 4}^{(1)}$ represents the land concentration score of the nth cell when its neighborhood (molar neighborhood) is a garden.

(2)Goal2: Competition among various types of land suitability The suitability of the land often reflects the degree of urban construction land suitability. In cities, the land suitability is determined by the slope and the urban forbidden construction area. In this study, the slope is divided into 5 levels, thus realizing the land. The quantification under the suitability goal, e while the urban forbidden construction area is a non-urbanized cell. In order to participate in the next data measurement.suitability in each competition situation is calculated by the decision goal matrix (2) combined with the upper limit effect measure, and the land affected by the land suitability can be calculated. Land types such as woodland, urban construction land and garden land are scored. The higher the score, the better the land suitability of the central cell, and the greater the possibility of conversion into urban construction land.

$$
\begin{aligned}
& u_{11}^{(2)} u_{12}^{(2)} u_{13}^{(2)} u_{14}^{(2)} u_{15}^{(2)} \\
& u_{21}^{(2)} u_{22}^{(2)} u_{23}^{(2)} u_{24}^{(2)} u_{25}^{(2)} \\
& r^{(2)}=\begin{array}{ccccc}
u_{31}^{(2)} & u_{32}^{(2)} & u_{33}^{(2)} & u_{34}^{(2)} & u_{35}^{(2)} \\
\vdots & \vdots & \vdots & \vdots & \vdots
\end{array}
\end{aligned}
$$

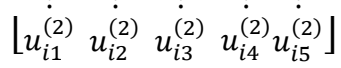

In the formula, $u_{\mathrm{n} 1}^{(2)}$ represents the land suitability score of the neighbor (moth neighborhood) of the nth cell if the land suitability is first grade, and $u_{\mathrm{n} 2}^{(2)}$ represents the nth cell. If the land suitability is a second-order land suitability score in the neighborhood (molar neighborhood), $u_{\mathrm{n} 3}^{(2)}$ represents the nth cell and its neighborhood (molar neighborhood) is when the land suitability is three. The land suitability score, $u_{\mathrm{n} 4}^{(2)}$, represents the land suitability score of the neighborhood (molar neighborhood) of the nth cell if the land suitability is four, and $u_{\mathrm{n} 5}^{(2)}$ represents the nth. Cell's neighbor (moth neighborhood) scores for land suitability when the land suitability is five.

(3) Goal 3: Traffic accessibility between different types of competition.

In the expansion of urban space, traffic accessibility plays a very important role. In the urban spatial expansion model, belt-like development along the traffic axis is more common. Therefore, there is a relatively high probability that the cells with high traffic development index will be converted into urban construction land. This study divides the equidistant buffers of urban highways into 
three levels, and then realizes quantification under traffic accessibility, which form a decision-making target matrix 3 , and then calculate the upper bound effect measure to calculate the traffic accessibility target. Three types of land (arable land, woodland, urban construction land) score. The higher the score, the better the traffic accessibility of the central cell, and the greater the possibility of conversion into urban construction land.

$$
r^{(3)}=\left[\begin{array}{ccc}
u_{11}^{(3)} & u_{12}^{(3)} & u_{12}^{(3)} \\
u_{21}^{(3)} & u_{22}^{(3)} & u_{23}^{(3)} \\
\vdots & \vdots & \vdots \\
u_{n 1}^{(3)} & u_{n 2}^{(3)} & u_{n 3}^{(3)}
\end{array}\right]
$$

In the formula $u_{\mathrm{n} 1}^{(3)}$ represents the traffic development score of the n-th cell whose neighbor (molar neighborhood) is a city construction site, and $u_{\mathrm{n} 2}^{(3)}$ represents the $\mathrm{n}$-th cell. If the neighborhood (Molar Neighbourhood) is the corresponding traffic development score for the cultivated land, $u_{\mathrm{n} 3}^{(3)}$ represents the traffic development score of the $n$-th cell when its neighborhood (molar neighborhood) is woodland.

(4) Goal 4: Competitiveness with distance constraints from the built-up area of the city center

In the expansion of urban space, the city center plays a key role in the development of the surrounding area. In urban space expansion mode, the pattern of growth along the center is more common. Therefore, there is a relatively high probability that urban cells with high radioactivity in urban centers will be converted into urban construction land. The study will buffer the distance from the built-up area of the city center and classify it into three levels, which is a schematic diagram of the local competition scenario at time t. It describes the decision targets formed under different central radiometers. The matrix, then combined with the upper limit effect measure, finally calculates the effect measure of the local class under the central radiometric target. The higher the score, the greater the radiation of the center cell by the city center and the greater the probability of conversion into urban construction land.

$$
r^{(4)}=\left[\begin{array}{ccc}
u_{11}^{(4)} & u_{12}^{(4)} & u_{12}^{(4)} \\
u_{21}^{(4)} & u_{22}^{(4)} & u_{23}^{(4)} \\
\vdots & \vdots & \vdots \\
u_{n 1}^{(4)} & u_{n 2}^{(4)} & u_{n 3}^{(4)}
\end{array}\right]
$$

In the formula, $u_{\mathrm{n} 1}^{(4)}$ represents the measurement value of the $\mathrm{n}$-th cell whose neighbor (molar neighbor) is a city center radiation degree, and $u_{\mathrm{n} 2}^{(4)}$ represents the $\mathrm{n}$-th cell. If the neighborhood (Molar Neighbourhood) is a measurement value when the central city's radiometric level is two, $u_{\mathrm{n} 3}^{(4)}$ represents the $\mathrm{n}$-th cell and its neighbor (Molar Neighbourhood) is the radius of the center of the city. It is a measure at the third level.

$$
r_{i j}^{(k)}\left\{\begin{array}{c}
u_{i j}^{(k)} / \max \left\{u_{i j}^{(k)}\right\} \\
u_{\min (i) \min (j)}^{(k)} / u_{i j}^{(k)} \\
u_{i_{0} j_{0}}^{(k)} / u_{i j}^{(k)}+\left|u_{i j}^{(k)}-u_{i j}^{(k)}\right|
\end{array}\right\}
$$

This is because of the above four constraints (competition among different types of cities under urban agglomeration, competition among different types of land under land suitability, competition among different types of localities under traffic accessibility, and competition under distance constraints from built-up areas of urban centers) The higher the effect measure, the better, so use the upper limit effect measure to calculate each single target, see equation (5). Each cell effect measure under different goals is expressed as:

$$
\begin{gathered}
S_{i}=\left[\frac{u_{i 1}^{(k)}}{s_{i 1}}, \frac{u_{i 2}^{(k)}}{s_{i 2}}, \cdots, \frac{u_{i n}^{(k)}}{s_{i n}}\right] \\
S_{j}=\left[\frac{u_{1 j}^{(k)}}{s_{1 j}}, \frac{u_{2 j}^{(k)}}{s_{2 j}}, \cdots, \frac{u_{m j}^{(k)}}{s_{m j}}\right]
\end{gathered}
$$

Then the four single-target results are combined to simulate the expansion of the urban space. After obtaining the results of the four single-target upper-bound effect measures, the formula (7) is used to calculate the comprehensiveness of land, farmland, garden land, urban construction land and other land types. The score, then compare the size of the value, take the maximum value as the optimal situation of the central cell, that is, the cell state at the next moment obtained after the four goals.

$$
\mathrm{M}^{(\mathrm{k})}=\left(\mathrm{r}_{\mathrm{ij}}^{(\mathrm{k})} / \mathrm{S}_{\mathrm{ij}}\right)_{\mathrm{n} \times \mathrm{m}}=\left[\begin{array}{cccc}
\frac{\mathrm{r}_{11}^{(\mathrm{k})}}{s_{11}} & \frac{\mathrm{r}_{12}^{(\mathrm{k})}}{\mathrm{s}_{12}} & \cdots & \frac{\mathrm{r}_{1 \mathrm{n}}^{(\mathrm{k})}}{\mathrm{s}_{1 \mathrm{n}}} \\
\frac{\mathrm{r}_{21}^{(\mathrm{k})}}{\mathrm{s}_{21}} & \frac{\mathrm{r}_{22}}{\mathrm{~s}_{22}} & \cdots & \frac{\mathrm{r}_{2 \mathrm{n}}}{\mathrm{s}_{2 \mathrm{n}}} \\
\cdots & \cdots & \cdots & \cdots \\
\frac{\mathrm{r}_{\mathrm{m}}^{(\mathrm{k})}}{\mathrm{s}_{\mathrm{m} 1}} & \frac{\mathrm{r}_{\mathrm{m}}^{(\mathrm{k})}}{\mathrm{s}_{\mathrm{m} 2}} & \cdots & \frac{\mathrm{r}_{\mathrm{mn}}^{(\mathrm{k})}}{\mathrm{s}_{\mathrm{mn}}}
\end{array}\right]
$$

In the formula, $r_{i j}^{(k)}$ is the comprehensive effect measure of the situation $S_{i j}$ under the K-objective; $u_{i j}^{(k)}$ is the actual effect under the k-th objective decision matrix; $\max \left(u_{i j}^{(k)}\right)$ The maximum effect in the effect of the k-th target decision matrix;

Then the four objectives (city agglomeration, urban land suitability, traffic accessibility, distance from the city center) and the single target effect measure and model parameters are calculated to obtain the multi-objective comprehensive effect measure size, see formula (8).

$$
r_{i j}^{\Sigma}=\sum_{k=0}^{\mathrm{n}} \omega_{k} r_{i j}^{(k)}
$$

Calculate the upper limit effect of $r_{i j}^{\Sigma}$ from 9, then the final multiobjective situation comprehensive decision matrix can be known from (9); then the combined effect measure of these neighbor cells is compared, and the results of different ranges are used as different situations. 


$$
M^{(\Sigma)}=\left(\frac{r_{i j}^{(\Sigma)}}{s_{i j}}\right)_{m \times n}==\left[\begin{array}{cccc}
\frac{r_{11}^{(\Sigma)}}{s_{11}} & \frac{r_{12}^{(\Sigma)}}{s_{12}} & \ldots & \frac{r_{1 n}^{(\Sigma)}}{s_{1 n}} \\
\frac{r_{21}^{(\Sigma)}}{s_{21}} & \frac{r_{22}^{(\Sigma)}}{s_{22}} & \ldots & \frac{r_{2 n}^{(\Sigma)}}{s_{2 n}} \\
\cdots & \cdots & \ldots & \cdots \\
\frac{r_{m 1}^{(\Sigma)}}{s_{m 1}} & \frac{r_{m 2}^{(\Sigma)}}{s_{m 2}} & \ldots & \frac{r_{m n}^{(\Sigma)}}{s_{m n}}
\end{array}\right]
$$

In the formula, $r^{(i)}$ represents the effect measure of the cell under the first goal decision matrix; $r_{i j}^{\left(\sum\right)}$ is the comprehensive effect measure of the central cell under multi-objective constraints; $\mathrm{P}$ is the model parameter (parameters of growth along the traffic line, urban agglomeration parameters, suitability parameters, radiation parameters of urban centers). Finally, compare the size of the four land types $r_{n 1}^{(\Sigma)}, r_{n 2}^{(\Sigma)}, r_{n 3}^{(\Sigma)}$, and $r_{n 4}^{(\Sigma)}$, and use the largest eigenvalue as $t+1$.

\subsection{Display of simulation results}

When the system model is simulated, it is assumed that the urbanized area will not change the type of land, and it is still the urban construction land. In addition, the non-buildable area is set as a static layer. The specific process of simulation is to first simulate the expansion results of urban space in 2005 using the 1998 data as the base year. This is the first simulation phase of the system. When the urban space expansion simulation system is used for simulation, it will have been preprocessed by the system. Post-1998 land classification status map and influencing factor layer data (traffic layer, gradient layer, non-buildable area layer, etc.) are loaded together, and the simulation results are adjusted in combination with the model parameters, and the iterative simulation results are iteratively The output image results are compared with the actual situation in 2005. When the results are basically consistent with 2005 , the optimal model parameters are output, and the next simulation is performed. Then, based on the 2005 land use status data as the base period data, the results of the simulation of urban space expansion in 2012 were taken as the second simulation stage. The urban spatial expansion simulation results in 2012 are shown in Figure 6;

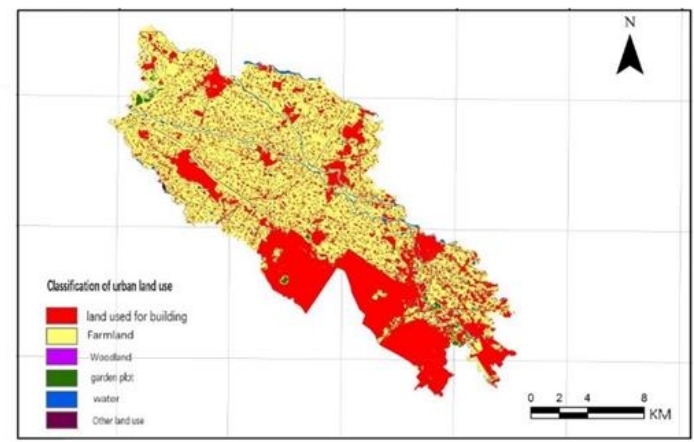

Figure 6 Comparison of simulation results for 2012

\subsection{Accuracy verification}

(1) Comparison between simulation results and actual results Comparing the actual number of urban land types and the number of simulated cells in Pidu District, Chengdu, 2012, it can be concluded that the greatest error is in forest land with an error of $5.27 \%$. The error in construction land, garden land, and arable land is normal, and the smallest error is cultivated land. Only $0.49 \%$. The spatial expansion model of the urban spatial model and the error of the actual current cell number are within the allowable range. The change trend is basically the same. As shown below.

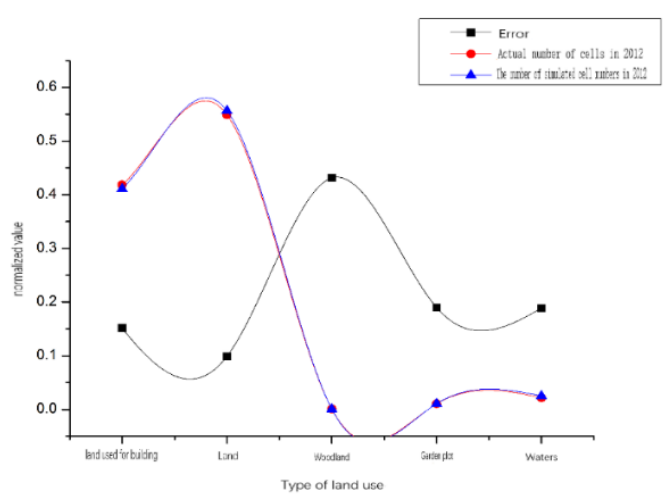

Figure7. Error Analysis of Simulation Results

\subsection{Model Forecast Results Display and Result Analysis}

Repeat the above simulation steps, based on 2005 data as the basic data and 2012 as the operational data, and then predict the urban spatial expansion layout of Chengdu City in 2019. The result is shown as:

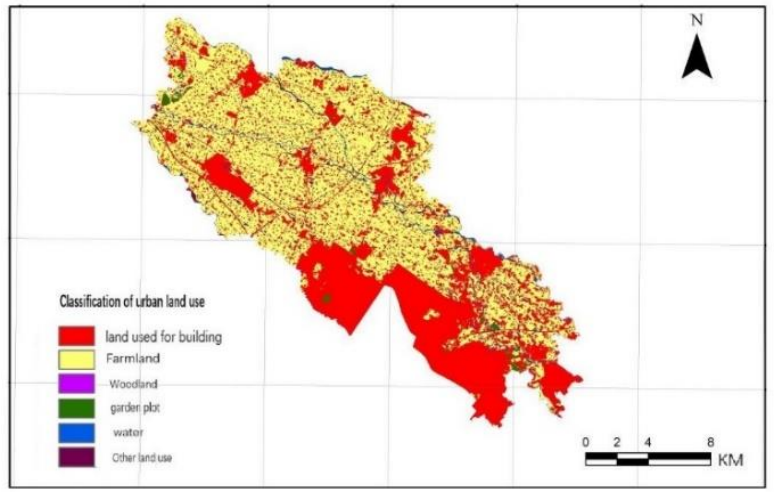

Figure 8 Forecast of Urban Land Use in 2019

2.5.1 Analysis of Spatial Distribution Changes:Based on the topological analysis of the base year and the 2019 forecast results in 2012, we can see from the comparison: (1) The builtup areas of the original urban centralized continuum are basically unchanged, while most of the increased urban construction land has a certain amount of The growth trend along the traffic line; (2) In the areas close to Chengdu City, such as Xihua School Front Area, Anjing Town, and some other areas, due to the effects of urban agglomeration and central radiation, some cultivated lands have been transformed into urban construction land, and the number of built-up areas has increased. The decentralized built-up area has decreased, indicating that there is a phenomenon of urban intensive development in Pidu District. This also has certain significance for the integration of Pidu District into the downtown area of Chengdu. (3) The relative changes in the structure of cultivated land, woodland, and gardens in the far suburbs of the northwestern area of Pidu District are relatively small, which is in line with the city's internal development law all along and has contributed to the development of the urban agricultural industry. 
Based on the above analysis, we study the phenomenon of regional urban spatial expansion. It can be seen that the changes in the district of Chengdu in the period of 2012-2019 are mainly reflected in two aspects: First, in the cities and towns near Chengdu's central city and in some areas around the traffic lines, the increase in construction land is more obvious; The internal transfer of land types is basically the conversion of cultivated land within the region to urban construction land, which is in line with the needs of intensive urban-intensive development.

2.5.2 Quantitative results analysis: The base period data of Pidu District in 2012 were compared with the forecast results of 2019 for the quantity of each class. It can be seen that: (1) The number of other non-urban construction land conversions to urban built-up areas has increased, with a rate of change of $2.3 \%$, of which urban construction land increased by nearly $12.6 \%$ and cultivated land types decreased by $5.7 \%$, reflecting urban construction land Most of the growth comes from the type of cultivated land; (2) The conversion rate of other non-urban land to other types of land use is $7.53 \%$, which is specifically the transformation of garden land and forest land.

It can be observed from the above results. First, the urban builtup areas in Chengdu's Pidu District are in line with the expansion of the original urban built-up areas. The expansion of urban land use has continued from the previous diversification model to intensive urban growth. In order to maintain a good momentum of urban growth, the government should strengthen forest land and gardens, especially urban afforestation, in the process of urban planning, and curb the continued occupation of forest land and gardens in the process of urban space expansion; Management and adjustment to avoid multiple competition between urban land and cultivated land; Second, in the 7 years between 2012 and 2019, urban construction land in Pidu District is growing steadily, and the overall level of land use is high. At the same time, the structural layout of other land types has changed little, which can promote the development of the agricultural industry in Farthern suburbs; Third, the comparative analysis of 1998, 2005, and 2012 shows that the urban land area predicted by 2019 is still in a continuous growth trend, indicating that for the Pidu District, which has superior geographic conditions and developed transportation routes, it is intensive. Urban expansion will be an evolutionary trend in the future. In general, during the period from 2012 to 2019, Pidu District was in a period of rapid urban development and was gradually improving. The area of natural semi-natural ecosystems such as cultivated land and gardens was reduced, and the area of urban construction land was increased. In the process of urbanization, Pidu District should focus on ecological city construction plans, supplemented by planning guidance and urban intensive plans, and focus on the development of cultivated land forestland quality in the northern and eastern parts of Pidu District. The southwest and southern regions need to be developed. Do a good job in the mid- and long-term urban development planning work, fully explore the city's internal land use, combine the scattered idle land within the city, and rationally prevent the disorder and fragmentation of the city. This will scientifically construct the urban landscape pattern and promote ecological civilization. Construction is of great significance.

\section{CONCLUSIONS AND PROSPECTS}

Based on the research of urban CA at home and abroad, this paper summarizes the research status and laws of urban space expansion. From the perspective of the neighborhood space, this paper proposes a model of urban space expansion based on a new combination of multi-objective gray decision and CA. An independent urban space expansion simulation system was developed in conjunction with the UML visual modeling language. Through the system, a case validation analysis was conducted. Based on the land classification data of Chengdu, in 1998, 2005, and 2012, the simulation was performed. The accuracy and validity of the model system were verified by the accuracy evaluation. The simulation prediction of urban space expansion in the Chengdu metropolitan area in 2019 was carried out. The main conclusions obtained are as follows:(1) This study proposes a new urban spatial expansion model, which uses a multi-objective grey decision-making method to tightly couple the city driving factors with the role of the neighborhood, and calculates the possibility of each cell expansion based on geographical conditions, traffic, and urbanization constraints. Sex. The model can take into account the neighborhood effect and the random characteristics of the urban cell itself to extract the intrinsic transition relationship of urban spatial expansion. It has a strong portability and flexibility for urban constraints. It solves the problem that the traditional cellular automata conversion rules can not meet the needs of the intrinsic spatiotemporal analysis of urban change, and overcomes the inconclusive problem of uncertainty in the coupling process between the urban construction driving factor and the urban cellular automata, which leads to the uncertainty of the simulation results. After simulation verification, the urban spatial expansion simulation error is less than $5.27 \%$, and the Kappa coefficient as a whole reaches 0.84 , indicating that the model can better capture the intrinsic transformation mechanism of the city. The use of this method is of great value for further excavation of ground driving principles and land development potential research.(2) Taking Pidu District of Chengdu City as an example, the urban land use status map is selected as the basic data of the simulation, and topographic data, traffic planning maps, and restrictions on construction land are used as urbanization control factors to simulate and predict the urban expansion status. The trend of regional urban expansion is to present a contiguous and contiguous urban growth model- "urban intensive development" model. This extended model is consistent with the theory of sustainable development and eco-urbanization design. It can also provide certain reference for the urban planning and construction of Pidu District, and it is of great significance for the government to formulate an effective land management policy.

\section{ACKNOWLEDGEMENTS}

This work was supported by National Key R\&D Program of China funded by ministry of Science and Technology of China (2017YFC1404705) and Natural Science Foundation of China (41171329).

\section{REFERENCES}

Duan Ruilan,Zheng Xinqi.2004.City simulation model (UrbanSim) and its application. Modern Urban Research, (01),pp.65-68.

Li Xia, Li Dan, Liu Xiaoping, Lao Chun Hua, Zhang Yihan, He Jinqiang, Huang Kangning.2010.GeoSOS software construction and application of geographic simulation optimization system.Acta Scientiarum Naturalium Universitatis Sun Yatseni, (04),pp.1-5+15.

Li Xia,Li Dan,Liu Xiaoping, Ho Jinqiang. 2009.Geographical simulation and optimization of GeoSOS and the frontier research system.Advances in Earth Science, (08),pp.899-907. 
Qian Min, Pu Li Jie and Zhu Ming. 2012.The land use change after the division of the main functional area based on the SLEUTH model.Transactions of the Chinese Society of Agricultural Engineering, 28(18),pp.223-232+294-295.

Sui, D. Z. and H. Zeng. 2001.Modeling the dynamics of landscape structure in Asia's emerging desakota regions: a case study in Shenzhen. Landscape and Urban Planning. 53,pp.37-52.

Wu F, Webster C J. 1998.Simulation of land development through the integration of cellular automata and multicriteria evaluation. Environment and Planning B: Planning and Design, 25(1),pp.103-126.

Waddell P. 2002.UrbanSim:Modeling urban development for land use,transportation, and environmental planning.Journal of the American planning association, 68(3) ,pp.297-314.

Wang Lei, Wang Yang, Cai Yunlong. 2012.Study on the ANNCA simulation of land use change -- take the cat jump river basin in the southwest Karst area as an example of.Acta Scientiarum Naturalium Universitatis Pekinensis, 48(01),pp.116-122.

Wu Wei, Zhou Sheng Lu, Wei Yehua,Liu Kehua, Chen Jianglong, Guan Weihua. 2013.Scenario simulation and spatial pattern evolution of urban growth in central urban area: a case study of Quanzhou, Fujian province. Geographical Research, 32(11),pp.2041-2054.

Xu Xi Bao, Yang Guishan, Zhang Jianming. 2009 Based on DUEM model, urban land use change in Lanzhou city.Arid Land Geography, 32(02) ,pp.289-295.

Xie Y. 1996.A generalized model for cellular urban dynamics. Geographical Analysis, 28(4),pp. 350-373. 